\title{
Étude comparative de quelques marqueurs de reformulation paraphrastique dans les articles de recherche et les articles de vulgarisation
}

Elsa Pic, Grégory Furmaniak et Vincent Hugou

\section{(2) OpenEdition \\ Journals}

Édition électronique

URL : http://journals.openedition.org/asp/3306

DOI : 10.4000/asp.3306

ISSN : 2108-6354

Éditeur

Groupe d'étude et de recherche en anglais de spécialité

Édition imprimée

Date de publication : 1 mars 2013

Pagination : 75-92

ISSN : 1246-8185

\section{Référence électronique}

Elsa Pic, Grégory Furmaniak et Vincent Hugou, «Étude comparative de quelques marqueurs de reformulation paraphrastique dans les articles de recherche et les articles de vulgarisation », ASp [En ligne], 63 | 2013, mis en ligne le 01 mars 2016, consulté le 03 novembre 2020. URL : http:// journals.openedition.org/asp/3306 ; DOI : https://doi.org/10.4000/asp.3306

Ce document a été généré automatiquement le 3 novembre 2020.

Tous droits réservés 


\title{
Étude comparative de quelques marqueurs de reformulation paraphrastique dans les articles de recherche et les articles de vulgarisation
}

\author{
Elsa Pic, Grégory Furmaniak et Vincent Hugou
}

\section{Introduction}

1 La problématique de la reformulation occupe une place centrale dans l'étude du discours de vulgarisation scientifique qui est un lieu hétérogène où circulent des discours sources, produits par des experts, et des discours seconds élaborés par des vulgarisateurs à l'adresse du grand public (Authier 1982 ; Mortureux 1982 ; Jacobi 1988 ; Beacco \& Moirand 1995 ; Reboul-Touré 2003). L'enjeu de la reformulation y est alors considéré comme stratégique, puisqu'il s'agit d'adapter son discours à un lectorat $a$ priori non avisé afin de l'informer, de l'instruire, de lui présenter les avancées d'une connaissance, ou encore de le distraire (Moirand et al. 1995).

2 Nous nous intéressons ici aux reformulations signalées par des connecteurs spécifiques et, plus précisément, aux reformulations auto-initiées, c'est-à-dire où l'auteur est à l'initiative de l'énoncé qu'il reformule (Gülich \& Kotschi 1983: 308). Avec Blandine Pennec (2006: 33), on peut poser qu'il y a reformulation paraphrastique lorsqu'il y a " équivalence, signalée par un marqueur, entre les contenus propositionnels de deux segments. Le second doit être considéré comme la ré-élaboration formelle du premier".

3 Cette étude s'inscrit dans le cadre d'un projet de recherche plus vaste intitulé «Sensibilité de la grammaire anglaise au degré de spécialisation» dont le principal objectif est de déterminer si le degré de spécialisation des textes, quelle que soit la 
discipline abordée, affecte leurs propriétés grammaticales (Pic \& Furmaniak 2010, 2012a, 2012b, 2012c). L'objectif du présent travail est de mener une analyse quantitative et qualitative des marqueurs de reformulation paraphrastique (désormais MRP). Au vu des études citées plus haut, on pourrait s'attendre à ce que le discours vulgarisé fasse plus grand usage des MRP, alors que le spécialiste s'adressant à ses pairs y aurait a priori moins recours. Mais nous allons montrer que les données ne confirment pas cette hypothèse et tenterons d'expliquer pourquoi.

Dans une première partie, nous présentons notre corpus et notre méthodologie. Nous livrons ensuite les premiers résultats quantitatifs dont le caractère inattendu nous amène à proposer une analyse qualitative des trois MRP dont la distribution varie grandement entre les deux sous-corpus, à savoir that is, i.e., et namely. Cette analyse s'appuie sur une classification des types de reformulation visant à mettre au jour les paramètres formels et fonctionnels pertinents pour expliquer le fonctionnement des trois MRP retenus.

\section{Méthodologie}

\subsection{Composition du corpus}

Les corpus existants ne convenant pas aux objectifs de notre projet, nous avons composé notre propre corpus, constitué d'articles de recherche extraits de revues à comité de lecture et d'articles de vulgarisation.

Chacune de ces deux sections, spécialisée et vulgarisée, est elle-même stratifiée en cinq sous-parties représentant cinq disciplines : la philosophie, l'histoire, l'économie, les mathématiques appliquées et l'astronomie. Chaque sous-corpus comptant 100000 mots, le corpus atteint un million de mots.

7 Les disciplines retenues sont variées afin que les spécificités dégagées puissent être attribuées au degré de spécialisation et non à une discipline ou à un groupement de disciplines.

8 Afin de limiter le parasitage dialectal et diachronique, n'ont été sélectionnés que des textes écrits par des auteurs britanniques entre 2000 et 2012. Nous avons veillé à ce que tous les textes soient écrits par des experts de la discipline. De cette façon, les variations linguistiques observées entre les deux types de discours ne peuvent être imputées à l'appartenance des auteurs à des groupes socio-culturels distincts mais à des facteurs pragmatico-rhétoriques liés à la prise en compte par l'auteur des connaissances supposées du lectorat ciblé.

9 Il convenait qu'au-delà des disciplines, les textes vulgarisés, d'un côté, et les textes spécialisés, de l'autre, s'adressent à des publics comparables. Le choix des publications s'est donc fondé sur la ligne éditoriale des sources considérées pour inclusion. Pour les textes spécialisés, nous avons retenu exclusivement des publications universitaires à comité de lecture reconnues (par exemple The Historical Journal, Cambridge Journal of Economics, Metaphilosophy, etc.) qui ont pour point commun d'être écrites par et pour des chercheurs. Les textes non spécialisés ont été sélectionnés dans des magazines de vulgarisation dite « experte » (Bondi 2012), c'est-à-dire une vulgarisation à destination d'un public parfois averti mais non expert et dont la ligne éditoriale témoigne d'une volonté d'atteindre le plus grand nombre, d'informer en restant accessible et de 
divertir. La présence de mots clés tels que "wide audience, accessible, general public, entertaining " dans la description des revues ou dans les conseils aux auteurs nous a paru être un indice suffisamment fort du niveau de vulgarisation pour motiver l'inclusion dans le corpus. Par exemple, History Today se présente comme " a unique cultural institution, bringing the best in historical writing and research to a wide audience»; Astronomy Now se réclame explicitement de la vulgarisation : «Astronomy Now falls into the genre of 'popular science magazine'" .

Enfin, nous avons fait le choix de sélectionner uniquement des textes complets, et non des extraits. Si les extraits augmentent la représentativité du corpus, ils rendent toutefois difficile la prise en compte de l'organisation interne spécifique des textes retenus. Or, ce type d'analyse de discours constitue une étape importante pour la suite du projet.

\subsection{Exploration du corpus}

11 Le corpus a été interrogé à l'aide du concordancier AntConc. L'extraction des reformulations paraphrastiques s'est faite à partir de requêtes spécifiques portant sur les marqueurs de reformulation suivants : that is, that is to say, i.e., in other words, namely, I mean et you mean. Cette liste est celle fournie par B. Pennec (2006) dont nous avons toutefois exclu or, en raison de son caractère polyfonctionnel (Tamba 1987) et de la ténuité des indices permettant de discriminer ses différents emplois. De trop nombreux exemples nous semblaient en effet problématiques et difficilement classables. C'est le cas de l'exemple (1) où le or peut aussi bien signaler une reformulation paraphrastique qu'un choix entre deux alternatives.

(1) He was given the name of Peter, but was variously known as 'Wild Peter', 'Peter

of Hanover', or, most famously, 'Peter the Wild Boy'. (VH) ${ }^{1}$

12 Cette recherche par marqueur s'est révélée productive, le taux de rappel étant très élevé. Néanmoins, un tri manuel des données est apparu nécessaire. Dans l'exemple (2), that est un pronom démonstratif. Le concordancier n'a bien évidemment pas permis de le mettre de côté.

(2) It was a conflict in which the investment bankers and traders generally came

out on top. That is, perhaps, the heart of the matter. (VE)

13 A été aussi éliminé, par exemple, l'énoncé (3) où le marqueur that is est antéposé par rapport à l'énoncé reformulé. Nous nous éloignons de l'ordre canonique <segment reformulé - marqueur - segment reformulant>.

(3) In contrast to the familiar philosophical thought that 'the true and real inner nature or kernel of man' is his 'knowing consciousness'-the thought, that is, that man is essentially a rational animal, or a soul, or res cogitans. (SP)

14 Au total, 483 occurrences de MRP ont été trouvées dans le corpus.

\section{Analyse quantitative}

\subsection{Différences disciplinaires}

Même si ce ne sont pas celles qui nous intéressent en priorité, il convient de faire état de différences importantes entre les disciplines en ce qui concerne l'emploi des MRP. 
On remarque tout d'abord, en observant la figure 1 et le tableau 1 , que les reformulations sont majoritaires en philosophie et en mathématiques appliquées.

Figure . Fréquence des MRP : différences disciplinaires

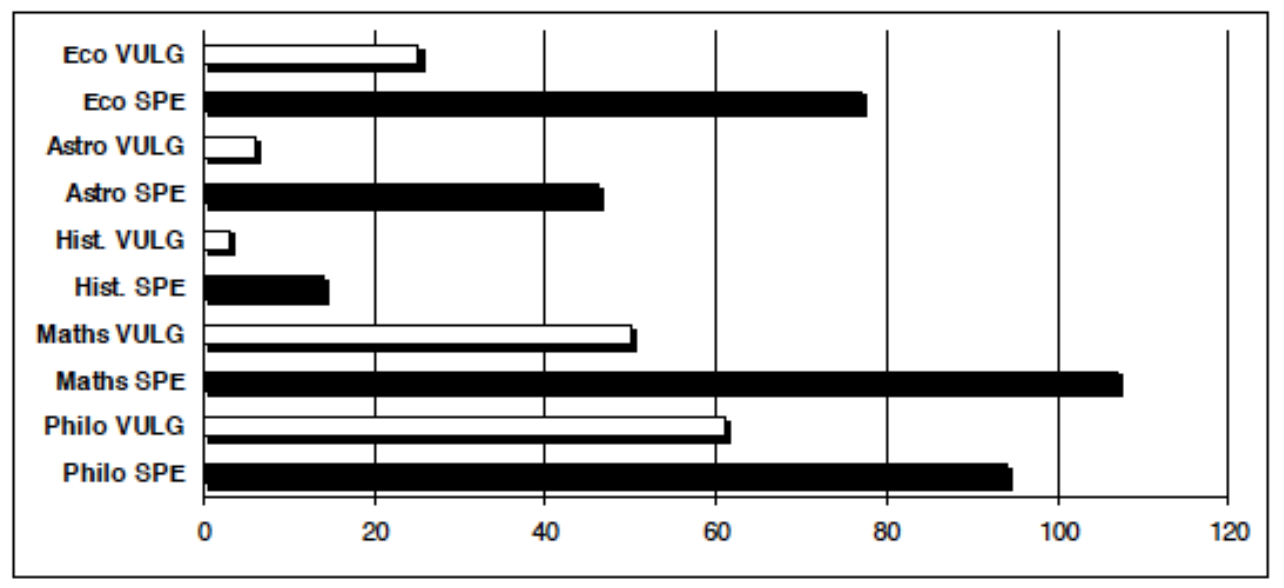

Tableau . Distribution des MRP dans les corpus spécialisé et vulgarisé

\begin{tabular}{|c|c|c|c|c|c|c|c|c|c|c|c|c|}
\hline & \multicolumn{4}{|c|}{ DISCOURS SPECIALISE } & \multicolumn{5}{c|}{ DISCOURS VULGARISE } \\
\cline { 2 - 16 } & Phllo & Maths & HIst & Astro & Eco & TOTAL & Phllo & Maths & HIst & Astro & Eco & TOTAL \\
\hline in other words & 12 & 7 & 1 & 2 & 15 & $\mathbf{3 7}$ & 4 & 24 & 1 & 7 & 9 & $\mathbf{4 5}$ \\
\hline that is & 26 & 25 & 3 & 0 & 15 & $\mathbf{6 9}$ & 18 & 10 & 1 & 0 & 6 & $\mathbf{3 5}$ \\
\hline that is to say & 4 & 0 & 2 & 1 & 2 & $\mathbf{9}$ & 5 & 3 & 0 & 0 & 1 & $\mathbf{9}$ \\
\hline namely & 16 & 22 & 3 & 7 & 8 & $\mathbf{5 6}$ & 11 & 4 & 0 & 4 & 5 & $\mathbf{2 4}$ \\
\hline i.e. & 36 & 53 & 5 & 36 & 37 & $\mathbf{1 6 7}$ & 23 & 8 & 1 & 6 & 4 & $\mathbf{4 2}$ \\
\hline I/you mean & 0 & 0 & 0 & 0 & 0 & $\mathbf{0}$ & 0 & 1 & 0 & 0 & 0 & $\mathbf{1}$ \\
\hline TOTAL & $\mathbf{9 4}$ & $\mathbf{1 0 7}$ & $\mathbf{1 4}$ & $\mathbf{4 6}$ & $\mathbf{7 7}$ & $\mathbf{3 3 8}$ & $\mathbf{6 1}$ & $\mathbf{5 0}$ & $\mathbf{3}$ & $\mathbf{1 7}$ & $\mathbf{2 5}$ & $\mathbf{1 5 6}$ \\
\hline
\end{tabular}

Notre travail ne porte cependant pas sur ce type de différences et le corpus, dans sa structuration même, n'a pas été conçu pour en rendre compte. La stratification en cinq disciplines vise en effet à rendre compte de la spécialisation et de la vulgarisation audelà des disciplines individuelles et permet de s'assurer que les résultats globaux ne sont pas faussés par une forte spécificité disciplinaire.

\subsection{Différences entre discours spécialisé et discours vulgarisé}

17 Ainsi, le tableau 1 et la figure 1 soulignent, au-delà des différences disciplinaires, trois phénomènes importants.

18 Tout d'abord, et contrairement à nos attentes, il apparaît que, globalement, l'expert écrivant un article de recherche utilise deux fois et demie plus de MRP que le vulgarisateur. La différence d'emploi des cinq premiers $\mathrm{MRP}^{2}$ discours spécialisé et discours vulgarisé est statistiquement significative $\left(X^{2}=36,566 ; d l=4 ; p<0,01\right)$.

Deuxièmement, la figure 1 montre assez clairement qu'en dépit des différences disciplinaires signalées plus haut, la fréquence d'emploi des MRP est supérieure dans le discours spécialisé au sein de chaque discipline : s'il y a, par exemple, plus de MRP en 
mathématiques vulgarisées qu'en histoire spécialisée, l'équilibre global à l'intérieur des sous-corpus de mathématiques et d'histoire reste inchangé.

Ce constat confirme les premières conclusions de Pic \& Furmaniak (2012c) qui ont en effet montré qu'il était possible de distinguer, entre discours spécialisé et discours vulgarisé, des propriétés distinctives relatives, mais non absolues.

Enfin, le tableau 1 fait apparaître que tous les MRP n'ont pas la même sensibilité au degré de spécialisation. À cet égard, la figure 2 est encore plus significative (le discours spécialisé est représenté par les colonnes noires, le discours vulgarisé par les colonnes blanches).

Figure . Distribution des différents MRP en corpus spécialisé et vulgarisé

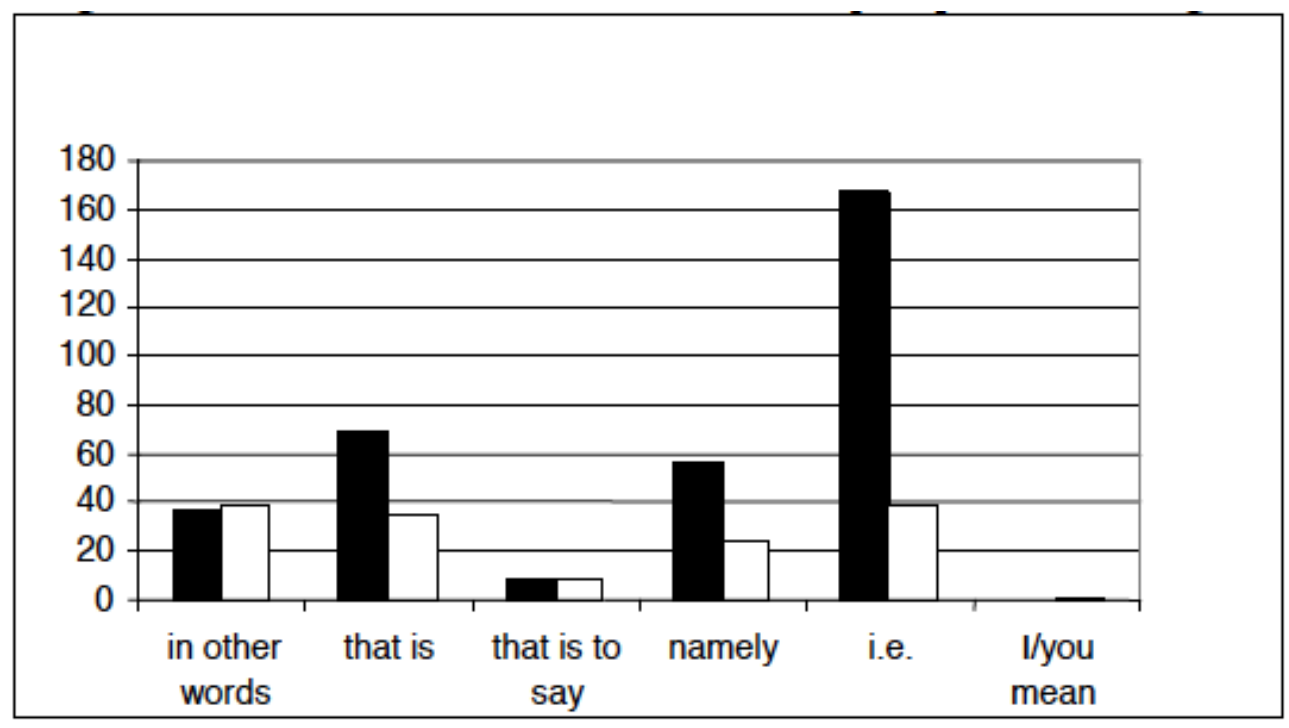

Les marqueurs in other words et that is to say sont ainsi répartis à parts égales entre le corpus spécialisé et le corpus vulgarisé. Il en va de même des MRP I mean et you mean qui, comme on pouvait s'y attendre dans un corpus écrit, n'apparaissent que très marginalement.

On note, en revanche, d'intéressantes différences de distribution entre le corpus spécialisé et le corpus vulgarisé concernant les marqueurs that is, i.e. et namely, dont la surutilisation dans les articles de recherche explique, quantitativement au moins, la tendance globale décrite plus haut. Ce sont ces trois MRP que nous allons maintenant étudier dans une perspective davantage qualitative, de sorte à compléter l'analyse quantitative qui ne suffit pas à rendre compte des phénomènes observés.

À cette fin, nous proposons une analyse du fonctionnement desdits MRP en fonction des deux paramètres que nous présentons ci-après et dont le niveau de granularité permet à la fois d'appréhender le fonctionnement des marqueurs et de mettre en relation leur emploi avec la fonction socio-pragmatique des deux genres étudiés.

\section{Paramètres d'analyse}

Le premier paramètre pertinent pour décrire le fonctionnement des MRP et pour justifier leur emploi dans un type de discours particulier a trait au registre des 
segments reformulés et reformulants. Le second critère concerne la fonction de la reformulation.

\subsection{Registre des segments : reformulation homogène ou hétérogène}

Ce critère d'analyse est emprunté à Pennec (2006).

\subsubsection{Reformulation hétérogène}

L'exemple ci-dessous est remarquable, car il contient une double reformulation :

(4) However, human life seems bereft of goodness only if we view it from without, that is, sub specie aeternitatis, in other words, in the view-from-nowhere of natural science. (VP)

Dans un premier mouvement, from without est reformulé par un syntagme adverbial appartenant à un registre plus savant et technique, sub specie aeternitatis. À son tour, ce syntagme est repris dans une langue plus courante par le marqueur in other words. Ce basculement d'un registre à un autre, quel qu'en soit le sens, sera qualifié de reformulation hétérogène.

\subsubsection{Reformulation homogène}

En (5), en revanche, le registre utilisé de part et d'autre du marqueur that is conserve une certaine homogénéité, puisqu'il est très technique dans les deux cas. On parlera de reformulation homogène.

(5) Thus we have 'gluing data' and given a perversity, that is, a pair $(\mathrm{m}, \mathrm{n}) \mathrm{Z} 2$ indexing $\mathrm{t}$-structures on $\mathrm{Dc}(\mathrm{U})$ and $\mathrm{Dc}(\mathrm{x})$, we can construct a $\mathrm{t}$-structure on $\mathrm{Dc}(\mathrm{X})$ [...]. (SM)

Notons qu'il peut exister des variations stylistiques au sein d'un même registre. En (5) par exemple, on passe d'une notion mathématique en langue naturelle (perversity) à son expression en langage formel (a pair $(m, n) Z 2$ indexing $t$-structures on $D c(U)$ and $D c(x)$ ). Toutefois, nous considérons qu'il s'agit dans les deux cas du même technolecte, d'où l'analyse en termes de reformulation homogène.

31 Cette définition restrictive de l'opposition homogène/hétérogène explique sans doute pourquoi nous n'avons pas rencontré de cas limites nécessitant la création d'une catégorie « indéterminé ».

\subsection{Fonction de la reformulation : clarifiante ou précisante}

\subsubsection{Reformulation clarifiante}

32 La seconde reformulation en (4) illustre ce que nous considérons comme la fonction prototypique des processus de reformulation. Il s'agit en effet de rendre transparente la formule latine potentiellement difficile d'accès. L'auteur anticipe une difficulté éventuelle de décodage et ajuste en conséquence son discours à son destinataire qui est ramené de ce qu'il ne connaît pas à ce qu'il connaît mieux. Il s'agit de dire différemment ou autrement pour clarifier et pour rendre plus accessible ce qui n'est pas supposé être clair, acquis ou stabilisé. 


\subsubsection{Reformulation précisante}

Il apparaît, par ailleurs, que le premier mouvement de reformulation en (4) ne va pas dans le sens d'une clarification. Au contraire, l'expression latine est opaque pour le lecteur néophyte et son emploi témoigne davantage d'un souci de précision et, peutêtre dans le même temps, de la volonté de la part de l'auteur de s'affirmer en tant qu'expert. Ce dernier trait est commun aux discours spécialisé et vulgarisé.

En discours vulgarisé, la reformulation précisante peut aussi permettre à l'auteur de se placer dans une visée didactique. En opérant du plus connu vers le moins connu, elle amène le lecteur vers un mot nouveau. Cependant, elle y est somme toute moins fréquente, car elle marque trop visiblement l'asymétrie de connaissances entre auteur et lecteur.

À l'inverse, elle est plus naturelle en discours spécialisé où la précision terminologique est un prérequis et où tout manquement dans ce domaine risquerait de porter atteinte à la «face positive » de l'auteur (Brown \& Levinson 1987), c'est-à-dire à sa réputation en tant qu'expert.

\subsection{Combinatoire}

Les deux facteurs pouvant se combiner, une reformulation paraphrastique peut donc théoriquement être :

- précisante et homogène ;

- précisante et hétérogène ;

- clarifiante et homogène ;

- clarifiante et hétérogène.

Cependant, certaines combinaisons sont moins naturelles et/ou limitées à certains contextes.

À présent, l'essentiel du travail consiste à déterminer dans quelle mesure et pourquoi certaines combinaisons sont plus fréquentes dans un corpus ou dans l'autre. Quels rôles jouent les marqueurs that is, i.e. et namely? Se caractérisent-ils par des différences d'emploi? Nous les analysons tour à tour, ce qui n'exclut pas des comparaisons ponctuelles.

\section{5. Étude des trois marqueurs sensibles au degré de spécialisation}

\subsection{Le marqueur that is}

\subsubsection{Caractéristiques générales}

Selon B. Pennec (2006: 90-105), that is institue une relation d'identité ou d'équivalence sémantique entre le segment reformulé et le segment reformulant. C'est un marqueur d'homogénéité stylistique et grammaticale entre ces deux segments. En (6), par exemple, le même code est utilisé, celui de la terminologie de la philosophie du langage (assertion/illocutionary), et la nature grammaticale (subordonnée de condition) est conservée. 
(6) It could only lead to EM if the relevant use is taken to be use in assertion or use in making a claim, that is, if the act is understood as illocutionary. (SP)

\subsubsection{Distribution de that is dans le corpus}

Le tableau 2, qui récapitule la distribution de that is au sein des deux sous-corpus, appelle un certain nombre d'observations.

Tableau . Distribution de that is dans le corpus

\begin{tabular}{|c|c|c|c|c|}
\hline & & & $\begin{array}{l}\text { DISCOURS } \\
\text { SPECIALISE }\end{array}$ & $\begin{array}{l}\text { DISCOURS } \\
\text { VULGARISE }\end{array}$ \\
\hline \multirow{6}{*}{ THAT IS } & \multirow{3}{*}{ Précisant } & Homogène & 51 & 14 \\
\hline & & Hétérogène & 2 & 1 \\
\hline & & Total & 53 & 15 \\
\hline & \multirow{3}{*}{ Clarifiant } & Homogène & 11 & 12 \\
\hline & & Hétérogène & 2 & 6 \\
\hline & & Total & 13 & 18 \\
\hline
\end{tabular}

Il ressort tout d'abord que si that is est surtout précisant, il peut également signifier une reformulation clarifiante.

e MRP est précisant dans l'exemple (7) où les « reformulations enchâssées » (Gülich \& Kotschi 1983) mettent en évidence l'intention de l'auteur de préciser son vouloir dire, et non le besoin de clarifier son propos.

(7) Interestingly, one natural way to understand Greco's proposal in this regard is as imposing some sort of sensitivity condition on knowledge. That is, knowledge results in cases where the agent has a true belief that is formed as a result of a reliable epistemic virtue which also meets the counterfactual condition that the true belief is sensitive to the truth, that is, if the proposition believed had not been true, then the agent would not have believed it in the way that she actually did [...]. (SP)

Avec that is, ces reformulations précisantes peuvent se décliner en explicitation de nombres, comme en (8), où l'auteur a pour souci de préciser les occurrences exactes des mises à jour; en recherche d'une dénomination juste, comme en (9), où il est question de mettre la «bonne étiquette » sur l'objet préalablement référé; ou en formalisation d'un concept, comme en (5), répété en (10), où la formalisation apparaît comme la façon la plus précise de décrire les phénomènes (surtout en mathématiques spécialisées et en philosophie spécialisée).

(8) The weights used for aggregating both indices are estimated using US Census data, updated every ten years, that is, in 1990, 2000, and 2010 [...]. (VE)

(9) However, it is the differential subfields of this set, that is, the Hardy fields, which are the focus of interest. (SM)

(10) Thus we have 'gluing data' and given a perversity, that is, a pair $(m, n) \mathrm{Z} 2$ indexing $\mathrm{t}$-structures on $\mathrm{Dc}(\mathrm{U})$ and $\mathrm{Dc}(\mathrm{x})$, we can construct a $\mathrm{t}$-structure on $\mathrm{Dc}(\mathrm{X})$ [...]. (SM)

Toutefois, that is peut également être utilisé à des fins de clarification, comme en (11).

(11) For instance, the tiling problem, that is, whether a given bunch of shapes will

tile a lane belongs to a class of mathematical problems called non-recursive. (VM)

Dans ce cas, la reformulation clarifiante peut consister en un exemple, comme en (12), ou en une définition, comme en (13). 
(12) [T] his is consistent with the fact that not all achievements are finally valuable. That is, perhaps some achievements, like wicked achievements, lack final value. (VP)

(13) Their structure is made up of two types of irregular polyhedra - a wonky looking dodecahedron with pentagonal faces (that is, it has twelve faces each with five sides) [...]. (VM)

L'exemple (13) s'apparente à une définition normée dont l'usage est plus justifié en vulgarisé. Il s'agit d'un mouvement qui va d'un technolecte au langage courant, au détriment parfois de la précision. Ce type de définition clarifiante est par conséquent à distinguer de la définition précisante, illustrée par (14), qui ne correspond plus vraiment à la définition communément admise, mais à la définition propre de l'auteur, celle qu'il impose, en quelque sorte, à son lecteur ${ }^{3}$.

(14) But what is true of language is true of everything else that is social (that is, everything whose existence depends on us). (SE)

La définition de social n'est pas celle du dictionnaire mais une définition orientée et subjective (whose existence depends on us) qui correspond à la propre perception de l'auteur.

En ce qui concerne le second paramètre (homogénéité/hétérogénéité), il ressort que that is est essentiellement un marqueur homogène. Ce trait est peut-être attendu en discours spécialisé : l'auteur utilise le même code (par exemple, le même technolecte) dans la mesure où il s'adresse à ses pairs, c'est-à-dire à d'autres experts du domaine. Point de nécessité, donc, de reformuler ses propos en changeant de code, ce qui pourrait, du reste, être vécu comme une atteinte à la face du lecteur et ce qui constitue pour l'auteur, comme on l'a vu supra, un moyen de préserver sa propre face.

C'est ce qui expliquerait la faible présence de that is dans le domaine de la vulgarisation scientifique, où il est question de se mettre à la portée d'un lecteur qui évolue dans une autre sphère discursive. Le trait « homogénéité » de that is limite alors son emploi dans ce type de discours: une reformulation de technolecte à technolecte n'est pas éclairante pour le néophyte. À l'inverse, une formulation en langage courant n'a pas a priori besoin d'être reformulée en langage courant, puisque le choix de ce registre est déjà motivé par un souci de transparence. Nous avons toutefois rencontré un énoncé curieux de reformulation du langage courant vers le langage courant. L'énoncé en (15) se rapproche de la tautologie :

(15) Thought and experience is possible for us only because we are limited and encounter difficulty, that is, things we bump up against. (VP)

\subsection{Le marqueur i.e.}

\subsubsection{Caractéristiques générales et distribution dans le corpus}

Selon B. Pennec (op. cit. : 129-35), le marqueur i.e. a de fortes connotations scientifiques et, à l'instar de that is, il établit une identité sémantique entre deux segments. C'est un marqueur homogène. Nos données confirment ces éléments.

51 Tout d'abord, la figure 3 montre qu'il existe en effet un fort déséquilibre entre le discours spécialisé et le discours vulgarisé, en faveur du spécialisé (164 occurrences dans les articles de recherche contre 37 dans les articles de vulgarisation). 
Figure . Distribution de i.e. en discours spécialisé et vulgarisé

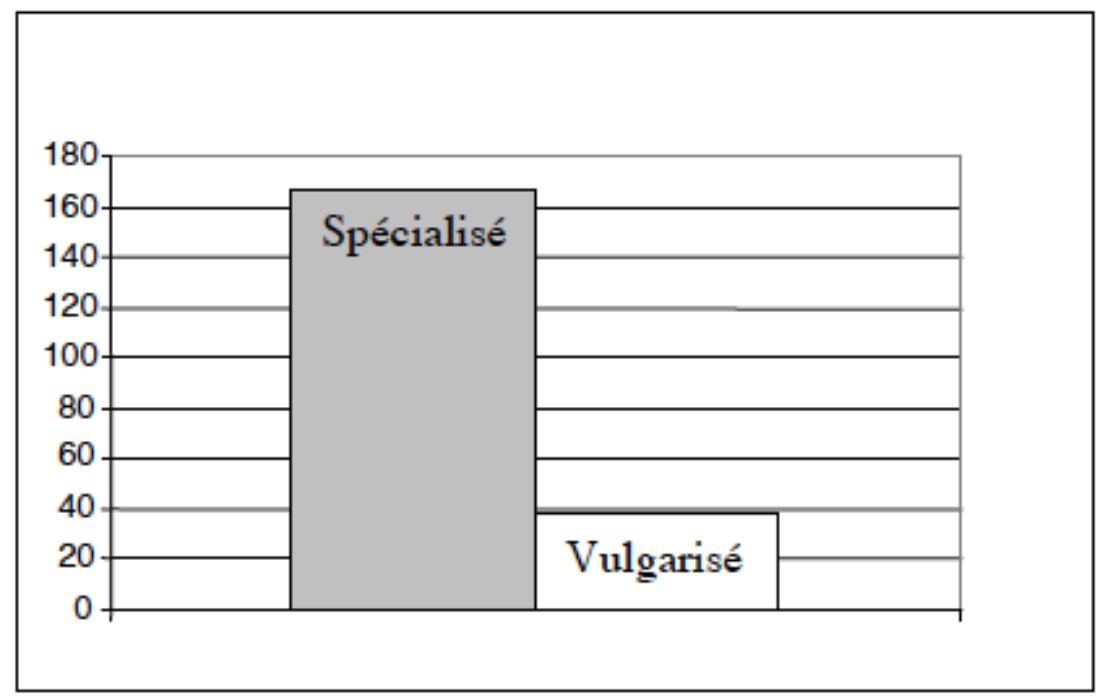

52 Ce résultat doit certes être nuancé : un texte de mathématiques spécialisées contient à lui seul trente-neuf occurrences de i.e. Pourtant, même en retranchant ce chiffre, la différence entre le spécialisé et le vulgarisé demeure considérable.

\subsubsection{Mise en regard des marqueurs i.e. et that is}

53 Les deux marqueurs présentant nombre de points communs, il est intéressant de traiter de i.e. en regard de ce qui vient d'être indiqué à propos de that is. Ils sont étymologiquement très proches, puisque id et that sont des pronoms démonstratifs anaphoriques et est et is correspondent aux formes conjuguées du verbe être.

Le tableau 3 montre que i.e., tout comme that is, est un MRP essentiellement précisant, ce qui explique sa surreprésentation en discours spécialisé. 
Tableau . Les différents paramètres pour i.e.

\begin{tabular}{|c|c|c|c|c|c|c|c|c|}
\hline & \multicolumn{6}{|c|}{ DISCOURS SPECIALISE } \\
\hline & & & Philo & Hist & Maths & Astro & Eco & Total \\
\hline \multirow{8}{*}{ بِّ } & \multirow[b]{3}{*}{ Précisant } & Homogène & 18 & 3 & 44 & 27 & 31 & 123 \\
\hline & & Hétérogène & 4 & 0 & 1 & 2 & 3 & 10 \\
\hline & & Total & 22 & 3 & 45 & 29 & 34 & 133 \\
\hline & \multirow[b]{3}{*}{ Clarifiant } & Homogène & 10 & 0 & 7 & 4 & 1 & 22 \\
\hline & & Hétérogène & 4 & 2 & 1 & 0 & 2 & 9 \\
\hline & & Total & 14 & 2 & 8 & 4 & 3 & 31 \\
\hline & \multicolumn{2}{|l|}{ Homogène } & 28 & 3 & 51 & 31 & 32 & 145 \\
\hline & \multicolumn{2}{|c|}{ Hétérogène } & 8 & 2 & 2 & 2 & 5 & 19 \\
\hline & & & \multicolumn{6}{|c|}{ DISCOURS VULGARISE } \\
\hline & & & Philo & Hist & Maths & Astro & Eco & Total \\
\hline \multirow{8}{*}{ بِّ } & \multirow[b]{3}{*}{ Précisant } & Homogène & 7 & 0 & 0 & 0 & 3 & 10 \\
\hline & & Hétérogène & 3 & 1 & 1 & 0 & 0 & 5 \\
\hline & & Total & 10 & 1 & 1 & 0 & 3 & 15 \\
\hline & \multirow[b]{3}{*}{ Clarifiant } & Homogène & 6 & 0 & 0 & 1 & 0 & 7 \\
\hline & & Hétérogène & 7 & 0 & 7 & 0 & 1 & 15 \\
\hline & & Total & 13 & 0 & 7 & 1 & 1 & 22 \\
\hline & \multicolumn{2}{|l|}{ Homogène } & 13 & 0 & 0 & 1 & 3 & 17 \\
\hline & \multicolumn{2}{|c|}{ Hétérogène } & 10 & 1 & 8 & 0 & 1 & 20 \\
\hline
\end{tabular}
i.e. Il peut, par exemple, être éclairant de faire une analogie comme dans (16) :

(16) This is the electromagnetic force (i.e. the photon jelly) acting on the electrons. of course the jelly is an analogy: there is a precise mathematical theory that describes it, and which talks about quantum fields instead of jelly. (VM)

À la lecture du tableau 3, on constate également que le MRP i.e. est de manière prépondérante un marqueur de reformulation homogène, comme that is, mais on observe quelques différences dans le sous-corpus vulgarisé où il semble pouvoir également fonctionner comme marqueur d'hétérogénéité, comme en (17) :

(17) J.L. Gaskin considers whether God's agency is like that of people who claim to be able to perform psychokinetic acts i.e. to make things happen without using their bodies. (VP)

Dans (17), l'auteur passe d'un terme technique à une périphrase définitionnelle qui s'appuie en partie sur l'étymologie du terme, à la manière d'une définition morphosémantique. On passe, par ailleurs, d'un terme d'origine gréco-latine (psychokinetic) à une reformulation en anglais.

Tableau . Comparaison des répartitions de that is et de i.e.

\begin{tabular}{|c|l|c|c|}
\cline { 3 - 4 } \multicolumn{2}{c|}{} & that is & i.e. \\
\hline \multirow{2}{*}{ DISCOURS SPECIALISE } & Homogène & 62 & 145 \\
\cline { 2 - 4 } & Hétérogène & 4 & 19 \\
\hline \multirow{2}{*}{ DIScours vulgarise } & Homogène & 26 & 17 \\
\cline { 2 - 4 } & Hétérogène & 7 & 20 \\
\hline
\end{tabular}

58 En raison de ses origines latines, le marqueur i.e. ne conserverait pas de manière «visible » la trace de son sens d'origine, alors que that forcerait le lecteur à garder en 
mémoire le référent repris de manière anaphorique. Formulé autrement, that is suppose clairement un mouvement de retour sur le dire et le dit. On peut donc penser que c'est le fonctionnement non explicitement anaphorique de i.e. qui lui confère, de temps à autre, en discours vulgarisé surtout, la possibilité de fonctionner comme un MRP hétérogène.

Il serait pourtant un peu hâtif de penser que les deux marqueurs, tous deux largement homogènes et précisants, sont parfaitement interchangeables. S'ils sont très proches sémantiquement et s'ils commutent facilement salva veritate, on note des différences stylistiques et syntaxiques. Dans certains cas, par exemple, that is occupe une position frontale, ce qui est syntaxiquement plus difficile pour i.e.

D'autre part, quelques différences disciplinaires, apparaissant dans le tableau 5, méritent également d'être examinées.

Tableau 5. Différences disciplinaires entre that is et i.e.

\begin{tabular}{|l|l|l|l|l|l|l|l|l|l|l|}
\hline & \multicolumn{4}{|l|}{ DISCours SPÉcIALISÉ } & \multicolumn{3}{|l|}{ DISCOURS VULGARISÉ } \\
\hline & Philo & Maths & Hist & Astro & Eco & Philo & Maths & Hist & Astro & Eco \\
\hline that is & 26 & 25 & 3 & 0 & 15 & 18 & 10 & 1 & 0 & 6 \\
\hline i.e. & 36 & 53 & 3 & 36 & 37 & 23 & 8 & 1 & 2 & 4 \\
\hline
\end{tabular}

61 En mathématiques spécialisées, i.e. est employé plus fréquemment que son pendant that is et, en astronomie spécialisée, aucune occurrence de that is n'est relevée. Au moins deux explications, non mutuellement exclusives, peuvent être avancées :

62 - l'utilisation d'une forme latine pourrait conférer aux travaux des auteurs un gage de scientificité. L'emploi des formules en mathématiques étant particulièrement développé, l'abréviation i.e. pourrait alors participer du même phénomène ;

63 - dans le discours scientifique, on peut supposer qu'un marqueur trop « voyant " crée un effet disruptif qui peut être mal perçu par le lecteur spécialiste. Le marqueur i.e., comme il a été précisé précédemment, est plus discret. On le rencontre d'ailleurs souvent à l'initiale de reformulations entre parenthèses, notamment en mathématiques spécialisées. Ce type d'intégration textuelle est intéressant pour le lecteur spécialisé, car il offre plusieurs niveaux de lecture. L'excès pouvant incommoder le lecteur averti, la mise entre parenthèses lui permet d'omettre la lecture d'un passage s'il estime que ce dernier ne lui apporte rien de nouveau.

\subsection{Le marqueur namely}

\subsubsection{Caractéristiques générales}

B. Pennec (op. cit. : 118-28) précise que namely est orienté vers la gauche, c'est-à-dire vers le segment reformulé et joue le rôle d'une complémentation sémantique, ce qui signale un rôle précisant dans notre typologie. Le marqueur est homogène.

On observe typiquement des cas de complémentation nominale avec possibilité de rétablir la construction <nom + subordonnée complétive>. Ces catégories nominales, 
parfois désignées par le terme de «shell-content constructions» (Schmid $2000: 4)$, sont toutes sous-spécifiées sémantiquement et c'est la subordonnée complétive qui leur donne leur épaisseur sémantique et qui en précise le sens. Bien plus que that is et i.e., l'emploi de namely est motivé par des raisons syntaxiques et de gestion de l'information.

Certaines occurrences dans notre corpus relèvent des mêmes analyses. On trouve essentiellement namely précédant des noms abstraits dénotant du dire ou du penser: implication, point, condition, premise, view, claim, question, assumption, etc. C'est par exemple le cas en (18), où la complétive that must be a single, fundamental or determining pattern [...], « introduite " par namely, précise le contenu de l'hypothèse mentionnée en début de phrase, tout en respectant deux principes fondamentaux de la gestion de l'information. En effet, les éléments syntaxiquement lourds ou complexes (par exemple, les subordonnées) et contenant de l'information nouvelle pour l'interlocuteur doivent figurer en fin de phrase ${ }^{4}$.

(18) One assumption built into Williams approach to this aspect of Wittgenstein's work is worth noting, and questioning, at the outset: namely, that there must be a single, fundamental or determining pattern of use of the we in Wittgenstein's later work. (SP)

67 Par ailleurs, B. Pennec (op. cit.) distingue deux fonctions pour le marqueur namely. Comme illustré en (19), il peut relier une désignation à la description de son référent. Cependant, il peut également mettre en rapport une désignation et une dénomination, comme en (20). Dans ce second cas de figure, les deux segments réfèrent strictement au même objet extralinguistique. La reformulation fournit la dénomination qui correspond au référent.

(19) [This] sheds critical light on the Catholic Church's success in mining one of the leading indicators of "true Americanism" in the post-World War II years, namely the branding of the Church itself with the pervasive Red excoriation of the era. (SH)

(20) Within the EU, defence R\&D is concentrated in six member states, namely France, Germany, Italy, Spain, Sweden and the UK, whose combined defence R\&D spending accounted for $99 \%$ of the EU. (VE)

\subsubsection{Distribution de namely dans le corpus}

En discours spécialisé comme en discours vulgarisé, namely sert d'abord à mettre en relation une désignation et une description, même si cette tendance est plus nette dans les articles de vulgarisation. Toutefois, cette différence de fonction fait surtout ressortir des différences disciplinaires (tableau 6). Comme pour les autres MRP étudiés, ce sont toujours les universitaires philosophes et leurs collègues mathématiciens qui y ont davantage recours, mais avec des fonctions différentes pour namely: le philosophe décrit alors que le mathématicien dénomme (des espaces, par exemple.) 
Tableau 6. Fonctions de namely

\begin{tabular}{|c|c|c|c|c|c|c|c|}
\hline & \multicolumn{6}{|c|}{ DISCOURS SPECIALISE } & \multirow[b]{2}{*}{$\%$} \\
\hline & Philo & Hist & Maths & Astro & Eco & Total & \\
\hline Dénomination & 2 & 1 & 15 & 3 & 4 & 25 & 44,6 \\
\hline \multirow[t]{2}{*}{ Description } & 14 & 2 & 7 & 4 & 4 & 31 & 55,4 \\
\hline & \multicolumn{6}{|c|}{ DISCOURS VULGARISE } & \\
\hline Dénomination & Philo & Hist & Maths & Astro & Eco & Total & $\%$ \\
\hline \multirow[t]{2}{*}{ Description } & 2 & 0 & 2 & 2 & 2 & 8 & 33,3 \\
\hline & 9 & 0 & 2 & 2 & 3 & 16 & 66,7 \\
\hline
\end{tabular}

69 Les résultats du tableau 7 montrent que namely est un marqueur d'homogénéité. En effet, $98 \%$ des reformulations contenant ce marqueur sont homogènes, quel que soit le sous-corpus.

Tableau 7. Distribution du marqueur namely (homogène/hétérogène)

\begin{tabular}{|c|c|c|c|c|c|c|}
\cline { 2 - 8 } \multicolumn{1}{c|}{} & \multicolumn{7}{c|}{ Discours SPECIALISE } \\
\cline { 2 - 8 } & Philo & Hist & Maths & Astro & Eco & Total \\
\hline Homogène & 15 & 3 & 22 & 7 & 8 & 55 \\
\hline Hétérogène & 1 & 0 & 0 & 0 & 0 & 1 \\
\hline & \multicolumn{7}{|c|}{ Discours vULGARISE } & Eco & Total \\
\cline { 2 - 8 } & Philo & Hist & Maths & Astro & 5 & 24 \\
\hline Homogène & 11 & 0 & 4 & 4 & 0 & 0 \\
\hline Hétérogène & 0 & 0 & 0 & 0 & & \\
\hline
\end{tabular}

70 De plus, comme l'indique le tableau 8, l'utilisation de namely est majoritairement à visée précisante en spécialisé et, dans une moindre mesure, en discours vulgarisé. Ces résultats étaient attendus étant donné la fonction de complémentation sémantique exposée supra.

Tableau 8. Distribution du marqueur namely (précisant/clarifiant)

\begin{tabular}{|c|c|c|c|c|c|c|}
\cline { 2 - 7 } \multicolumn{1}{c|}{} & \multicolumn{7}{c|}{ Discours SPECIALISE } \\
\cline { 2 - 8 } \multicolumn{1}{c|}{} & Philo & Hist & Maths & Astro & Eco & Total \\
\hline Précisant & 15 & 3 & 22 & 7 & 8 & $\mathbf{5 5}$ \\
\hline Clarifiant & 1 & 0 & 0 & 0 & 0 & $\mathbf{1}$ \\
\hline & \multicolumn{7}{|c|}{ Discours vulGARISE } \\
\cline { 2 - 8 } & Philo & Hist & Maths & Astro & Eco & Total \\
\hline Précisant & 8 & 0 & 4 & 4 & 5 & $\mathbf{2 1}$ \\
\hline Clarifiant & 3 & 0 & 0 & 0 & 0 & $\mathbf{3}$ \\
\hline
\end{tabular}

71 Ce MRP, à l'instar de that is et de i.e., est donc lui aussi majoritairement précisant et homogène. C'est la combinaison de ces deux traits dans les trois MRP étudiés qui explique donc leur sensibilité au degré de spécialisation des textes ainsi que leur emploi privilégié dans les articles de recherche. Les traits "clarifiant " et "hétérogène » constitueraient la combinaison attendue pour les MRP propres au discours de vulgarisation, mais aucun marqueur n'a révélé cette composition, expliquant sans doute le fait que nous n'avons pu identifier de MRP spécifiques aux articles de vulgarisation. 


\section{Conclusion}

L'exploration du corpus pluridisciplinaire de langue spécialisée et de langue vulgarisée a permis de dégager des tendances dans le domaine de la reformulation paraphrastique avec marqueurs spécifiques. Sur le plan quantitatif, contrairement aux attentes initiales, il est apparu que le spécialiste faisait un plus grand usage des MRP que le vulgarisateur. Trois marqueurs sensibles au degré de spécialisation des textes, that is, i.e. et namely, ont fait l'objet d'une attention plus particulière. Sur le plan qualitatif, une typologie a été établie pour rendre compte de ces répartitions. Les reformulations relevées dans notre corpus ont été classées en fonction de leur visée précisante ou clarifiante ainsi qu'en fonction du degré d'homogénéité du code de part et d'autre du MRP. D'une manière générale, le discours spécialisé utilise des reformulations précisantes : il s'agit le plus souvent pour l'auteur de rendre accessible son vouloir dire afin d'asseoir son raisonnement, de donner sa propre interprétation, de nuancer son propos rétroactivement ou même de prévenir des inférences indésirables. Le dessein est tout autre en discours vulgarisé où la reformulation précisante peut procéder d'un souci d'instruire le lecteur tout en donnant au vulgarisateur, selon son niveau d'expertise sans doute, l'occasion de renvoyer une image valorisante de lui-même.

Le travail peut être encore approfondi en étudiant, par exemple, les reformulations extra-discursives qui mettent en jeu les propos d'autres experts, ce qui engage une réflexion sur les citations et sur le discours rapporté. Notre analyse gagnera également à être croisée avec celle des modes de discours qui sont l'une des spécificités du projet. En s'inspirant des travaux d'Adam (1992) et de Smith (2003), une typologie fine des modes de discours a en effet été établie (Pic \& Furmaniak 2012c). Le travail d'annotation discursive est en cours et ne permet pas encore d'obtenir des résultats statistiquement signifiants, mais il sera intéressant de vérifier si certains types de reformulations s'inscrivent de manière préférentielle dans certains modes de discours.

\section{BIBLIOGRAPHIE}

Adam, Jean-Michel. 1992. Les Textes : types et prototypes. Paris : Nathan.

Authier, Jacqueline. 1982. «La mise en scène de la communication dans des discours de vulgarisation scientifique ». Langue française 53, 34-47.

Beacco, Jean-Claude et Sophie Moirand. 1995. « Autour des discours de transmission des connaissances ». Langages 117, 32-53.

Bondi, Marina. 2012. "Re-contextualizing knowledge. Past and present in popular history". Communication au 4th International 360 Conference "Encompassing Knowledge Mediation". Aarhus, Danemark, 10-12 mai 2012.

Brown, Penelope et Stephen Levinson. 1987. Politeness: Some Universals in Language Use.

Cambridge : Cambridge University Press. 
Gülich, Elizabeth et Thomas Kotschi. 1983. «Les marqueurs de la reformulation paraphrastique ». Cahiers de linguistique française 5, 305-51.

Jacobi, Daniel. 1986. Diffusion et vulgarisation : itinéraires du texte scientifique. Paris : Les Belles Lettres.

Jacobi, Daniel. 1988. «Le discours de vulgarisation : problèmes sémiotiques et textuels ». In Jacobi, D. et B. Schiele (dir.), Vulgariser la science - le procès de l'ignorance. Seyssel : Champ Vallon, 87-117.

Khatchatourian, Elisaveta. 2008. « Les marqueurs de la reformulation formés à partir du verbe dire ». In Le Bot, M.-C. (dir.), Marqueurs linguistiques : stratégies énonciatives. Rennes : Coll. Rivages, Presses Universitaires de Rennes, 19-33.

Moirand, Sophie, A. Ali Bouacha, J.-C. Beacco et A. Collinot (dir.). 1995. Parcours linguistiques de discours spécialisés. Berne : Lang.

Mortureux, Marie-Françoise. 1982. « Paraphrase et métalangage dans le discours de vulgarisation ». Langue française 53, 48-61.

Pennec, Blandine. 2006. « La reformulation en anglais contemporain : indices linguistiques et constructions discursives ». Thèse de Doctorat, Université Rennes 2.

Pic, Elsa et Grégory Furmaniak. 2010. « Grammaire et degré de spécialisation ». ASp 58, 39-55.

Pic, Elsa et Grégory Furmaniak. 2012a. « De la langue spécialisée à la langue vulgarisée : approche grammaticale ». ASp 61, 39-54.

Pic, Elsa et Grégory Furmaniak. 2012b. « A study of epistemic modality in academic and popularised discourse: The case of possibility adverbs perhaps, maybe and possibly ». Revista de Lenguas para Fines Específicos 18, 11-44.

Pic, Elsa et Grégory Furmaniak. 2012c. «Les modes de discours, interface entre texte et grammaire dans les langues de spécialité ». ASp 62, 25-44.

Reboul-Toure, Sandrine. 2003. « La glose entre langue et discours ». Langues et langage 9, 75-91. Reboul-Toure, Sandrine. 2004. «Écrire la vulgarisation scientifique aujourd'hui ». Colloque Sciences, Médias et Société, 15-17 juin 2004, Lyon, ENS-LSH.

Schmid, Hans-Jörg. 2000. English Abstract Nouns as Conceptual Shells. From Corpus to Cognition. Berlin : Mouton de Gruyter.

Smith, Carlota. 2003. Modes of Discourse. The local Structure of Texts. Cambridge : Cambridge University Press.

Tamba, Irène. 1987. « 'Ou' dans les tours du type : un bienfaiteur public ou un évergète ». Langue française 73, 16-28.

\section{NOTES}

1. Le marqueur de reformulation est en gras pour faciliter la lecture. Pour la source des exemples, nous adoptons la convention qui suit. La première lettre indique le sous-corpus (Spécialisé ou Vulgarisé), la seconde lettre, la discipline (Philosophie, Histoire, Astronomie, É conomie ou Mathématiques appliquées).

2. L'absence d'occurrence de I/you mean en discours spécialisé nous oblige à exclure ce MRP pour le calcul du chi-carré. 
3. Khatchatourian (2008: 26) parle d'« emploi d'auteur ».

4. Il s'agit des principes du end-weight et du end-focus.

\section{RÉSUMÉS}

Cette étude est menée dans le cadre d'un projet visant à mettre au jour les spécificités grammaticales des articles de recherche et des articles de vulgarisation. Il s'agit de montrer que si, comme l'ont exposé de nombreux auteurs, vulgarisation et reformulation en tant qu'activités discursives sont effectivement étroitement associées, cette corrélation fonctionnelle n'implique pas pour autant une surutilisation des marqueurs explicites de reformulation dans les textes vulgarisés. Trois marqueurs (en particulier « that is, i.e. » et " namely ») sont clairement propres aux articles de recherche. Pour rendre compte de ce résultat, nous dégageons deux paramètres, l'un formel, l'autre fonctionnel, dont les valeurs se combinent pour générer quatre grands types de reformulation: hétérogène clarifiante, hétérogène précisante, homogène clarifiante et homogène précisante. Ainsi, il apparaît que chacun des deux discours étudiés privilégie, en raison de sa visée socio-pragmatique, certaines combinaisons, tandis que chacun des marqueurs de reformulation est employé prioritairement dans un ou deux types de reformulation, ce qui explique leur fréquence dans les articles de recherche et leur relative rareté en discours vulgarisé.

This study is part of a wider research project which seeks to describe the grammatical properties of research articles and popular science articles. As many authors have shown, there is a close connection between reformulation and popularization understood as discourse practices. However, this does not entail that writers of popular science make extensive use of formal markers of reformulation. In fact, the corpus analysis shows that three connectors ("that is, i.e." and "namely") are specific to research articles. We suggest that two parameters are relevant to account for this: (i) Is the reformulation homogeneous or heterogeneous? (ii) Is it intended as a means of making things clearer or more precise? Each genre, because of its socio-pragmatic properties, is associated with one or two kinds of reformulation. The same holds of individual markers, which explains why they are used frequently in research articles and much less so in popular science articles.

\section{INDEX}

Mots-clés : article de recherche, i.e., namely, reformulation paraphrastique, that is, vulgarisation

Keywords : i.e., namely, popularization, reformulation, research article, that is 


\section{AUTEURS}

\section{ELSA PIC}

Agrégée d'anglais, Elsa Pic est maître de conférences en linguistique anglaise à l'Université Sorbonne Nouvelle - Paris 3. Ses recherches portent sur la terminologie, l'anglais juridique et les spécificités linguistiques des langues de spécialité. elsa.pic@univ-paris3.fr

\section{GRÉGORY FURMANIAK}

Agrégé d'anglais, Grégory Furmaniak est maître de conférences en linguistique anglaise à l'Université Sorbonne Nouvelle - Paris 3. Il a une longue expérience de l'enseignement en secteur Lansad. Ses recherches portent sur l'aspect et la modalité en synchronie et en diachronie, ainsi que sur l'anglais de spécialité. gregory.furmaniak@univ-paris3.fr

\section{VINCENT HUGOU}

Agrégé d'anglais, Vincent Hugou est PRAG et doctorant en linguistique anglaise à l'Université Sorbonne Nouvelle - Paris 3. Il effectue une partie de ses cours dans le secteur Lansad. Ses recherches portent sur la lexicologie ainsi que sur l'anglais de spécialité. vincent.hugou@univparis3.fr 